\title{
COMPORTAMENTO DA CV. PÉROLA (Phaseolus vulgaris L.) SUBMETIDA A DIFERENTES NÍVEIS DE DESFOLHA ARTIFICIAL ${ }^{1}$
}

\author{
MURILO FAZOLIN ${ }^{2}$ \\ JOELMA LIMA VIDAL ESTRELA ${ }^{3}$
}

\begin{abstract}
RESUMO - Estudos sobre desfolha artificial são muito úteis, principalmente para simular danos às plantas, como os causados por insetos ou granizo, por exemplo. Em trabalhos com o feijoeiro, ficou evidente que os resultados são altamente dependentes da cultivar utilizada e das condições climáticas prevalecentes. Objetivou-se com este trabalho avaliar a redução na produtividade devida à redução artificial da área foliar da cv. Pérola nas condições de Rio Branco, AC. O experimento foi conduzido no campo experimental da Embrapa Acre, em delineamento de blocos casualizados, com quatro repetições e esquema experimental de parcelas subdivididas, estudando-se nas parcelas cinco das etapas de desenvolvimento da planta (V3= primeira folha trifoliolada, V4= terceira folha trifoliolada, R6= floração, R7= formação de vagens e R8= enchimento de vagens) e nas
\end{abstract}

subparcelas, quatro níveis de desfolhamento $(0 \%, 33 \%$, $66 \%$ e 100\%). Foram avaliadas, em média, 480 plantas nas 4 linhas centrais de cada subparcela, determinandose o número de vagens por planta, o número de grãos por vagem e a produtividade de grãos. Paralelamente, em uma área adjacente, foram colhidas, ao acaso, 50 plantas de cada etapa de desenvolvimento para a obtenção da área foliar média. Nas etapas de desenvolvimento V3, V4 e R7, níveis de desfolhamento a partir de $33 \%$ causaram decréscimo no número de vagens por planta. $\mathrm{O}$ número de sementes por vagem não sofreu influência dos níveis de desfolhamento das plantas. O rendimento dos grãos foi significativamente reduzido à medida que as plantas foram submetidas a níveis crescentes de desfolha. A etapa de florescimento (R6) foi a que apresentou maior redução na produtividade como resposta à desfolha.

TERMOS PARA INDEXAÇÃO: Feijão comum, etapas de desenvolvimento, perda de produtividade.

\section{EFFECT OF DIFFERENT LEVELS OF DEFOLIATION ON PRODUCTIVITY OF COMMON BEANS (Phaseolus vulgaris L. CV. PEROLA)}

\begin{abstract}
Studies to evaluate artificial defoliation are very useful, mainly to simulate damages to plants, such, as the ones caused by insects or hail. Experiments with bean plant, indicated that the results are higly dependent on the cultivars used, and preponderant climatic conditions. The purpose of this study was to evaluate yeld reduction due to the reduction on the leaf area at different stages of growth of bean crop of Perola cultivar under environmental conditions of Rio Branco, Acre. The experiment was conducted at the Experimental Station of Embrapa Acre. The experiment was arranged as a split-plot in a randomized block design, with four replications. The main plots consisted of five plant development stages: $\mathrm{V} 3=$ first trifoliate leaf, $\mathrm{V} 4=$ third trifoliate leaf, $\mathrm{R} 6=$ initiation of flowering , $\mathrm{R} 7=$ pod formation and $\mathrm{R} 8=$ pod
\end{abstract}

maturation, and the sub-plots were the four defoliation levels $(0 \%, 33 \%, 66 \%$ and 100\%). At harvest an average of 480 plants of the four central lines of each sub-plot were evaluated. It was determined the number of pods per plant, number of seeds per pod and yeld $(\mathrm{kg} / \mathrm{ha})$. In order to determine the average leaf area, 50 plants were randomily harvested in each development stage. The results demonstrated that defoliation levels starting from 33\%, in the stages V3, V4 and R7, reduced the number of pods per plant. The number of seeds per pod was not affected by the level of defoliation of the plants. The seed weight was significantly reduced as the plants were submitted to increasing levels of defoliation. The flowering stage (R6) presented the highest yeld reduction with defoliation of the plants.

INDEX TERMS: Common bean, development stages, reduction in productivity.

1. Trabalho financiado pelo Banco da Amazônia S.A. Basa.

2. Doutor em Entomologia - Pesquisador da Embrapa Acre.

3. Mestre em Entomologia - Bolsista CNPq/DCR. 


\section{INTRODUÇÃO}

Inúmeros são os trabalhos de pesquisa sobre desfolhamento artificial em leguminosas de importância econômica, como soja e feijão, desenvolvidos com o objetivo de simular danos causados por insetos ou chuvas de granizo. Nesses trabalhos, fica evidenciado que há divergências entre os resultados obtidos, o que pode estar relacionado com as diferentes metodologias empregadas (Higley, 1992), e, principalmente, com a influência do meio ambiente. Hohmann e Carvalho (1982) e Moura e Mesquita (1982), por exemplo, observaram maiores reduções na produção do feijoeiro quando o desfolhamento foi realizado no início da formação das vagens. Bortoli et al. (1982) afirmaram que o estádio mais crítico foi o florescimento, ao passo que Galvez et al. (1977) concluíram que não há estádio mais crítico, e sim um período que vai da floração ao enchimento de vagens.

Apesar de a cv. Pérola ser uma das mais plantadas no Brasil (Yokoyama et al., 1999) e representar, em média, 32,5\% do total de sementes básicas de feijão produzidas no País, existem poucos trabalhos que avaliaram a influência do desfolhamento sobre o comportamento da cv. Pérola, talvez por acreditar-se que essa cultivar apresenta o mesmo comportamento de outras cultivares já avaliadas. No entanto, Galvez et al. (1977), Chagas et al. (1979), Santa Cecília et al. (1980) e Link et al. (1980) mencionam que esse tipo de estudo deve ser regionalizado, uma vez que há diferenças no comportamento das cultivares, em função das condições ambientais em cada região.

Quintela e Barrigossi (1999), avaliando níveis de desfolha artificial da cv. Pérola em cultura irrigada no Brasil Central, constataram que 50\% de desfolha quando as plantas apresentam as folhas primárias, $30 \%$ no estádio vegetativo e $15 \%$ de desfolha na fase reprodutiva são indicativos para a adoção de medidas de controle, a fim de não causar prejuízos econômicos.

No Acre, a principal praga desfolhadora do feijoeiro é a Cerotoma tingomarianus Bechyné (Coleoptera: Chrysomelidae), que ataca a cultura desde o início do desenvolvimento das plantas, podendo atingir níveis de consumo foliar de até $80 \%$ durante as etapas de florescimento e maturação de vagens (Fazolin e Silva, 1996). Foi observado por Fazolin et al. (2001) que nas pulverizações com inseticidas visando ao controle dessa praga não são levados em conta, pelos pequenos agricultores do Acre, os critérios técnico-econômicos. Isso resulta em conseqüências danosas ao meio ambiente, devido ao número excessivo de aplicações, muitas delas realizadas com produtos não registrados para a cultura do feijoeiro e em etapas de desenvolvimento da cultura que não justificam o procedimento.

Conduziu-se este trabalho com o objetivo de avaliar o comportamento da cv. Pérola perante a redução artificial da área foliar em várias etapas de desenvolvimento das plantas, para gerar informações básicas para o MIP de C. tingomarianus, nas condições do estado do Acre.

\section{MATERIAL E MÉTODOS}

O experimento foi conduzido na safra agrícola de 2001, em Argissolo Vermelho, no campo experimental da Embrapa Acre, situado à latitude de 9० 58' 29" e longitude de $67^{\circ} 48^{\prime} 36^{\prime}$, , com clima do tipo AM, de acordo com a classificação de Köppen, ou seja, quente e úmido, com estações seca e chuvosa bem definidas (Oliveira e Alvarenga, 1985).

A cultivar de feijão Pérola foi semeada no espaçamento $0,50 \mathrm{~m}$ entre linhas, com população aproximada de 200.000 plantas/ha, conforme recomendação de Valério et al. (1999). O delineamento estatístico foi o de blocos casualizados com quatro repetições, em esquema de parcelas subdivididas. As parcelas foram constituídas por cinco das etapas de desenvolvimento do feijoeiro (Santos e Gavinales, 1998): V3 (primeira folha trifoliolada), V4 (terceira folha trifoliolada), R6 (floração), R7 (formação de vagens) e R8 (enchimento de vagens). As subparcelas corresponderam a quatro níveis de desfolhamento $(0 \%, 33 \%, 66 \%$ e $100 \%)$. Cada subparcela foi composta de seis linhas de $4 \mathrm{~m}$ de comprimento, sendo avaliadas, em média, 480 plantas das 4 linhas centrais $\left(24 \mathrm{~m}^{2}\right)$.

Os desfolhamentos foram efetuados quando $50 \%$ das plantas da área útil atingiram as etapas de desenvolvimento desejadas. Para se obter os níveis estabelecidos, retirou-se, de todas as folhas, com o auxílio de uma tesoura, o número de folíolos correspondente às porcentagens de desfolhamento das plantas.

Com a finalidade de controlar as pragas, foram realizadas, a intervalos de três dias, pulverizações com produto à base de carbaryl.

Por ocasião da colheita, todas as plantas da parcela útil foram contadas, determinando-se ainda o número de vagens por planta, o número de sementes por vagem e o peso das sementes de toda a parcela.

Em uma área de $450 \mathrm{~m}^{2}$, adjacente ao experimento, foram coletadas, ao acaso, 50 plantas em cada etapa de desenvolvimento avaliada. Uma vez desfolhadas e preparadas, foram submetidas à leitura digitalizada da área foliar, utilizando o software PCXAREA, segundo Wilcken et al. (1995), obtendo-se, assim, a área 
foliar média produzida em cada etapa de desenvolvimento.

Os dados obtidos foram submetidos à análise de variância. Os efeitos das etapas de desenvolvimento foram avaliadas pelo teste de médias de Scoot e Knott (1974), enquanto os efeitos dos níveis de desfolha foram avaliados por meio da análise de regressão.

\section{RESULTADOS E DISCUSSÃO}

Avaliando-se individualmente os diferentes níveis de desfolha da cv. Pérola de feijoeiro, não foi constatada diferença significativa na produção de vagens entre as etapas de desenvolvimento (Tabela 1). Isso significa que cada nível de desfolha permitiu a mesma produção de vagens, independente da etapa de desenvolvimento em que foi aplicada ao feijoeiro.

No entanto, a análise de regressão mostrou que a resposta da produção de vagens nos diferentes níveis de desfolha foi diferenciada em cada etapa de desenvolvimento (Figura 1).
TABELA 1 - Número médio de vagens por feijoeiro da cv. Pérola submetida a quatro níveis de desfolhamento, em diferentes etapas de desenvolvimento.

\begin{tabular}{ccccc}
\hline \multirow{2}{*}{$\begin{array}{c}\text { Etapas de } \\
\text { senvolvimento* }\end{array}$} & \multicolumn{4}{c}{ Níveis de Desfolha (\%) } \\
\cline { 2 - 5 } & $\mathbf{0}$ & $\mathbf{3 3}$ & $\mathbf{6 6}$ & $\mathbf{1 0 0}$ \\
\hline V3 & $9,2 \mathrm{a}$ & $6,8 \mathrm{a}$ & $6,6 \mathrm{a}$ & $5,2 \mathrm{a}$ \\
V4 & $9,0 \mathrm{a}$ & $6,4 \mathrm{a}$ & $6,2 \mathrm{a}$ & $4,4 \mathrm{a}$ \\
R6 & $6,6 \mathrm{a}$ & $5,6 \mathrm{a}$ & $5,0 \mathrm{a}$ & $4,2 \mathrm{a}$ \\
R7 & $8,4 \mathrm{a}$ & $6,4 \mathrm{a}$ & $6,0 \mathrm{a}$ & $3,8 \mathrm{a}$ \\
R8 & $7,4 \mathrm{a}$ & $6,4 \mathrm{a}$ & $4,8 \mathrm{a}$ & $4,6 \mathrm{a}$ \\
\hline MÉDIA & 8,12 & 6,32 & 5,72 & 4,44 \\
\hline
\end{tabular}

*V3= primeira folha trifoliolada, $\mathrm{V} 4=$ terceira folha trifoliolada, $\mathbf{R 6}=$ floração, $\mathbf{R 7}=$ formação de vagens $e$ $R 8=$ enchimento de vagens.

Médias seguidas de mesma letra nas colunas não diferem significativamente entre si pelo teste de Scott e Knott $(P<0,05) C V=19,76 \%$.

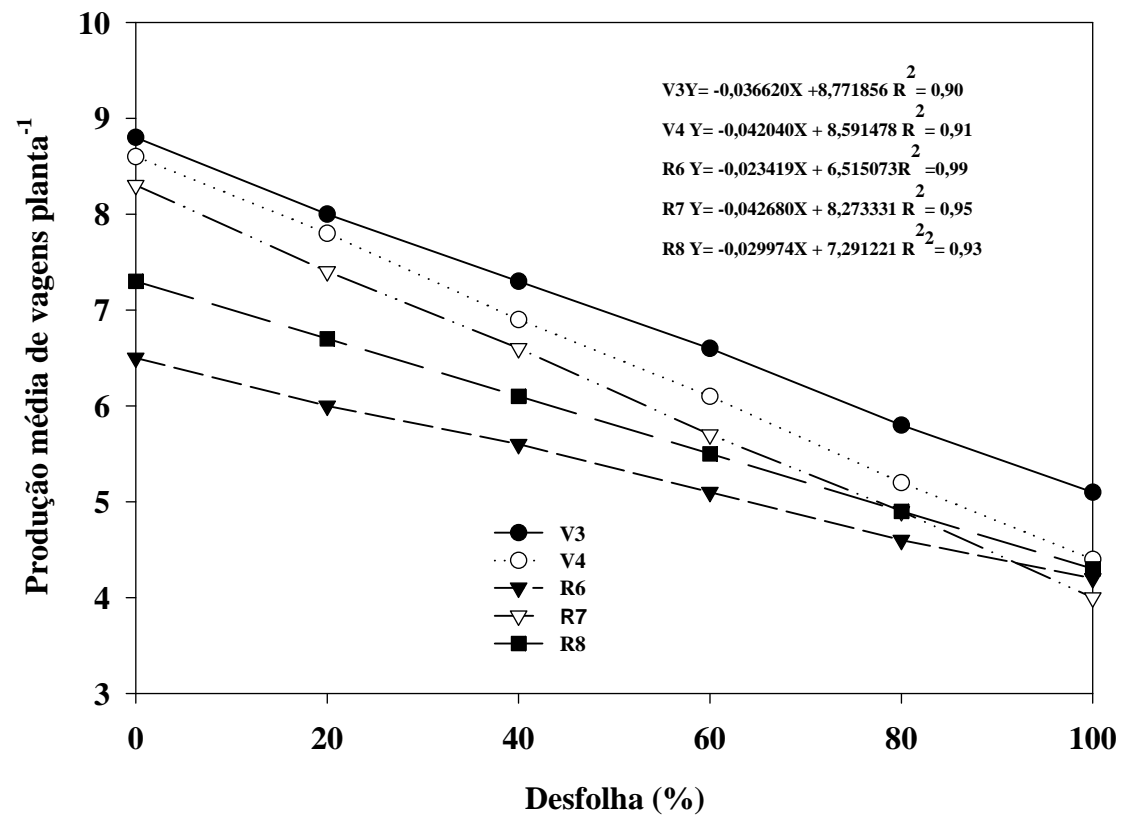

FIGURA 1 - Redução na produção de vagens por planta do feijoeiro cv. Pérola em função dos níveis de desfolhamento e etapas de desenvolvimento das plantas: V3= primeira folha trifoliolada, $\mathrm{V} 4=$ terceira folha trifoliolada, $\mathrm{R} 6=$ floração, $\mathrm{R} 7=$ formação de vagens e R8= enchimento de vagens. 
Quando as plantas foram desfolhadas nas etapas iniciais do desenvolvimento vegetativo (V3 e V4) e na etapa de formação de vagens (R7), apresentaram decréscimo acentuado no número de vagens, o que ficou evidenciado pelos baixos coeficientes angulares negativos das retas, quando comparados com os das etapas de floração (R6) e enchimento de vagens (R8).

Quintela e Barrigossi (1999), na etapa R6, não observaram diferença significativa no número de vagens produzidas pelos níveis de desfolhamento de $33 \%$ e $66 \%$ nessa mesma cultivar. No entanto, nas condições de Rio Branco, os níveis de desfolhamento a partir de $66 \%$ provocaram queda acentuada na produção de vagens nas etapas de florescimento (R6) e enchimento de vagens (R8). Os maiores coeficientes angulares das retas, nessas duas etapas, podem sugerir maior tolerância das mesmas ao desfolhamento crescente, quanto à produção de vagens.

Independente da etapa de desenvolvimento considerada, a menor produção de vagens/planta foi observada no desfolhamento de $100 \%$.

Independente do nível de desfolha, não houve diferença significativa entre as etapas de desenvolvimento das plantas quanto ao número de sementes produzidas por vagem (Tabela 2). Também para essa variável não houve significância dos níveis de desfolha e nem da in- teração nas etapas de desenvolvimento x níveis de desfolha.

TABELA 2 - Número médio de grãos por vagem da cv. Pérola de feijoeiro submetida a quatro níveis de desfolhamento em diferentes etapas de desenvolvimento.

\begin{tabular}{ccccc}
\hline $\begin{array}{c}\text { Etapas de } \\
\text { Desenvolvimen- } \\
\text { to* }\end{array}$ & \multicolumn{4}{c}{ Níveis de Desfolha (\%) } \\
\cline { 2 - 5 } & $\mathbf{0}$ & $\mathbf{3 3}$ & $\mathbf{6 6}$ & $\mathbf{1 0 0}$ \\
\hline V3 & $2,2 \mathrm{a}$ & $2,2 \mathrm{a}$ & $2,2 \mathrm{a}$ & $2,6 \mathrm{a}$ \\
V4 & $2,6 \mathrm{a}$ & $2,4 \mathrm{a}$ & $2,6 \mathrm{a}$ & $4,0 \mathrm{a}$ \\
R6 & $2,6 \mathrm{a}$ & $2,6 \mathrm{a}$ & $2,4 \mathrm{a}$ & $2,4 \mathrm{a}$ \\
R7 & $3,0 \mathrm{a}$ & $3,0 \mathrm{a}$ & $3,0 \mathrm{a}$ & $3,2 \mathrm{a}$ \\
R8 & $2,8 \mathrm{a}$ & $3,2 \mathrm{a}$ & $3,2 \mathrm{a}$ & $3,0 \mathrm{a}$ \\
\hline MÉDIA & 2,64 & 2,68 & 2,68 & 3,04 \\
\hline
\end{tabular}

*V3= primeira folha trifoliolada, V4= terceira folha trifoliolada, $\mathrm{R6}=$ floração, $\mathrm{R} 7=$ formação de vagens $\mathrm{e}$ $R 8=$ enchimento de vagens.

Médias seguidas de mesma letra nas colunas não diferem significativamente entre si pelo teste de Scott e Knott $(\mathbf{P}<0,05) \quad$ CV $=27,79 \%$.

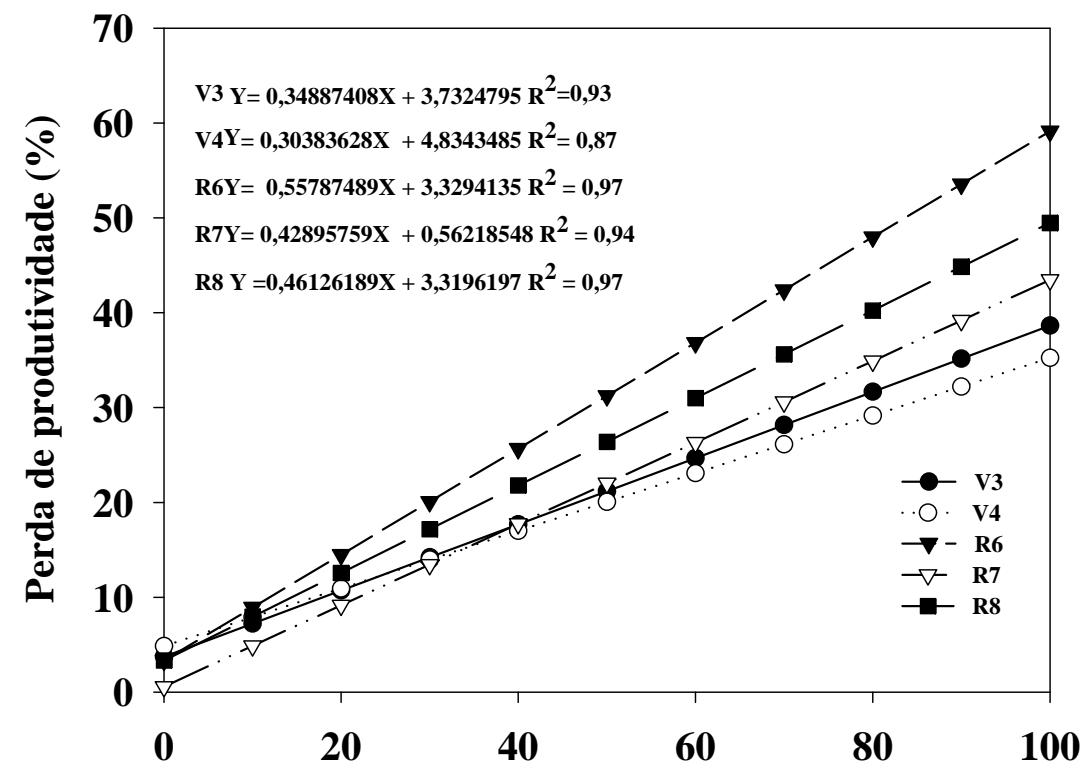

Desfolha (\%)

FIGURA 2 - Redução na produtividade do feijão cv. Pérola em função dos níveis de desfolhamento e etapas de desenvolvimento das plantas: V3= primeira folha trifoliolada, V4= terceira folha trifoliolada, R6=floração, R7= formação de vagens e R8= enchimento de vagens. 
A perda de área foliar afetou visivelmente o peso de grãos, o que se traduziu em conseqüente queda de produtividade (Figura 2). Resultados semelhantes foram obtidos por Bortoli et al. (1982) e Hohmann e Carvalho (1982), os quais observaram que, além do número de vagens, o peso dos grãos é reduzido em função da perda de área foliar.

Avaliando-se individualmente cada nível de desfolha da cv. Pérola de feijoeiro, não foi constatada diferença significativa de produtividade entre as etapas de desenvolvimento (Tabela 3). Isso significa que, considerando-se cada nível de desfolha de forma independente, a produtividade foi semelhante em todas as etapas de desenvolvimento.

TABELA 3 - Produtividade média $\left(\mathrm{kg} \mathrm{ha}^{-1}\right)$ de feijoeiro cv Pérola submetido a quatro níveis de desfolhamento em diferentes etapas de desenvolvimento.

\begin{tabular}{ccccc}
\hline \multirow{2}{*}{$\begin{array}{c}\text { Etapas de } \\
\text { Desenvolvimento* }\end{array}$} & \multicolumn{5}{c}{ Níveis de Desfolha (\%) } \\
\cline { 2 - 5 } & $\mathbf{0}$ & $\mathbf{3 3}$ & $\mathbf{6 6}$ & $\mathbf{1 0 0}$ \\
\hline V3 & $1.546 \mathrm{a}$ & $1.232 \mathrm{a}$ & $1.135 \mathrm{a}$ & $984 \mathrm{a}$ \\
V4 & $1.465 \mathrm{a}$ & $1.223 \mathrm{a}$ & $1.144 \mathrm{a}$ & $1.055 \mathrm{a}$ \\
R6 & $1.465 \mathrm{a}$ & $1.124 \mathrm{a}$ & $960 \mathrm{a}$ & $646 \mathrm{a}$ \\
R7 & $1.694 \mathrm{a}$ & $1266 \mathrm{a}$ & $1.197 \mathrm{a}$ & $838 \mathrm{a}$ \\
R8 & $1.602 \mathrm{a}$ & $1.454 \mathrm{a}$ & $1.118 \mathrm{a}$ & $871 \mathrm{a}$ \\
\hline MÉDIA & $1.554,4$ & $1.244,1$ & $1.126,7$ & 879,0 \\
\hline
\end{tabular}

*V3= primeira folha trifoliolada, $\mathrm{V} 4=$ terceira folha trifoliolada, R6=floração, $R 7=$ formação de vagens e $\mathbf{R 8}=$ enchimento de vagens.

Médias seguidas de mesma letra nas colunas não diferem significativamente entre si pelo teste de Scott e Knott $(\mathrm{P}<0,05) . \mathrm{CV}=\mathbf{2 0 , 1 8 \%}$.

A redução linear na produtividade devida ao desfolhamento, em cada etapa de desenvolvimento, indica que a cv. Pérola é sensível às perdas foliares em qualquer etapa de desenvolvimento da planta (Figura 2). Esse resultado deverá subsidiar o cálculo do nível de ação para o controle de $C$. tingomarianus, levando-se em consideração as variáveis descritas por Nakano et al. (1981), para essa finalidade.

Durante as etapas de desenvolvimento vegetativo V3 e V4 até a formação de vagens (R7), as plantas apresentaram reduções médias de produtividade de $20,0 \%, 27,5 \%$ e 43,5\%, respectivamente, com os níveis de desfolhamento de $33 \%$, 66\% e $100 \%$. Já na etapa de enchimento de vagens (R8), a redução na produtividade somente foi acentuada a partir de $66 \%$ de desfolha, constatando-se, portanto, que nessa etapa as folhas remanescentes, mesmo em altos níveis de desfolha, são suficientes para a síntese de fotoassimilados necessários para a produção de grãos. Resultados semelhantes foram obtidos em soja, por Moura e Costa (1981) e Pissaia e Costa (1981).

Quintela e Barrigossi (1999) não observaram redução na produtividade do feijoeiro quando a desfolha se deu durante as etapas vegetativas V3 e V4, ocorrendo redução significativa no rendimento $(65 \%)$ somente quando as plantas foram submetidas à desfolha total durante o período compreendido entre a etapa de florescimento (R6) e enchimento de vagens (R8). Esses resultados não foram confirmados no presente trabalho em Rio Branco, uma vez que em R6 ocorreu acentuada redução de produtividade, correspondendo a $27,7 \% ; 38,2 \%$ e $58,4 \%$, respectivamente, nos níveis de desfolha de 33\%, 66\% e $100 \%$. Quintela e Barrigossi (1999) observaram ainda que as desfolhas causaram maiores reduções no rendimento quando realizadas na etapa de formação de vagens (R7), em comparação ao florescimento (R6).

Por ser tratar de cultivar de hábito de crescimento indeterminado entre os tipos II e III (porte semi-ereto a prostrado), a cv. Pérola mostrou alternância nos valores de área foliar produzida durante as etapas de desenvolvimento (Figura 3). Da etapa V3 para a etapa V4, ocorreu um incremento acentuado na emissão de novas folhas $\left(205,81 \mathrm{~cm}^{2}\right.$ planta $\left.{ }^{-1}\right)$, refletindo em menores reduções de produtividade, mesmo quando submetida a altos níveis de desfolha (acima de 66\%). Na etapa R6, o incremento foi de apenas $74,07 \mathrm{~cm}^{2}$ planta $^{-1}$, justificando a importância da área foliar produzida nessa etapa, conforme ressaltado por Portes (1996), já que, $80 \%$ dos fotoassimilados permanecem nas folhas e tecidos circunvizinhos. Pelos resultados obtidos por Jones (1971), aponta-se que o rendimento de Phaseolus depende do índice foliar durante o florescimento, porque se a haste principal e os ramos laterais funcionarem de maneira autônoma, maior crescimento dos ramos resultará em aumento de área foliar, maior interceptação de luz e, conseqüentemente, em maior produtividade de matéria seca e de grãos. No presente estudo, essa maior dependência da área foliar produzida em R6 ficou evidenciada pela maior inclinação da reta correspondente a essa etapa, refletindo em maiores perdas de produtividade (Figura 2), sugerindo que essa etapa de desenvolvimento é a mais sensível ao desfolhamento, em comparação com as demais. 


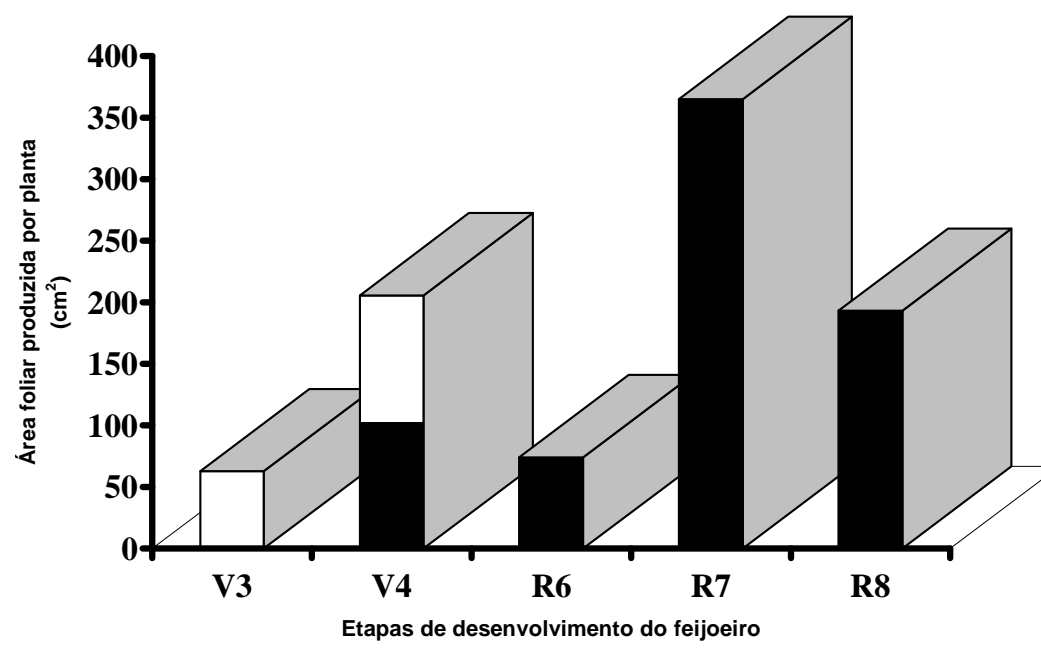

\section{$\mathrm{V} 3=$ primeira folha trifoliolada, $\mathrm{V} 4=$ terceira folha trifoliolada, $R 6=$ floração, $R 7=$ formação de vagens e $R 8=$ enchimento de vagens.}

FIGURA 3 - Área foliar produzida em cada etapa de desenvolvimento do feijoeiro cv. Pérola.

Para a cultivar Pérola, entretanto, essa dependência descrita por Jones (1971) não parece restrita à etapa R6, uma vez que na etapa da formação de vagens (R7) as plantas retomaram a emissão de folhas, apresentando o maior valor de área foliar $\left(365,24 \mathrm{~cm}^{2}\right.$ planta $\left.{ }^{-1}\right)$ observado, compensando, de certa forma, as perdas de produtividade (vide a menor inclinação da reta da Figura 2). Esse comportamento pode implicar em vantagem para as plantas dessa cultivar, uma vez que $45 \%$ dos fotoassimilados sintetizados pelas folhas medianas, nessa etapa, são translocados para as vagens, da mesma forma que $75 \%$ das folhas dos ramos são direcionados para a formação de grãos no mesmo ramo (Portes, 1996). Talvez essa seja a razão da queda significativa de produtividade observada a partir de $66 \%$ de desfolhamento nas etapas R7 e R8.

\section{CONCLUSÕES}

Nas etapas de desenvolvimento V3, V4 e R7, níveis de desfolhamento a partir de 33\% causaram decréscimo no número de vagens por planta.

O número de sementes por vagem não sofreu influência dos níveis de desfolhamento das plantas. O rendimento dos grãos foi significativamente reduzido à medida que as plantas foram submetidas a níveis crescentes de desfolha.
A etapa de florescimento (R6) foi a que apresentou maior redução na produtividade como resposta à desfolha.

\section{AGRADECIMENTOS}

Ao laboratorista Valdemir de Souza e Silva e ao assistente de pesquisa Charles Rodrigues da Costa, da Embrapa Acre, pela contribuição e empenho na condução dos experimentos.

\section{REFERÊNCIAS BIBLIOGRÁFICAS}

BORTOLI, S. A. de; NAKANO, O.; PERECIN, D. Efeito de desfolhas e dobras artificiais de folíolos sobre a produtividade do feijoeiro (Phaseolus vulgaris L.) em cultivo das águas. Científica, São Paulo, v. 10, n. 1, p. 105-111, 1982.

CHAGAS, J. M.; VIEIRA, C.; MAESTRI, M.; CARDOSO, A. A. Resposta de duas variedades de feijão (Phaseolus vulgaris L.) ao desfolhamento artificial. Ciência e Cultura, São Paulo, v. 6, n. 31, p. 683-687, 1979.

FAZOLIN, M.; PESSOA, J. de S.; AMARAL JÚNIOR, D. L. do; OLIVEIRA, W. S. A. de.; COSTA, C. R. da. Determinação do nível de ação para o controle da vaquinha-do-feijoeiro no Acre. Rio Branco: Embrapa, 2001. 4 p. (Comunicado Técnico, 134). 
FAZOLIN, M.; SILVA, W. S. da. Níveis de danos e comportamento populacional de Cerotoma tingomarianus Bechyné, na cultura do feijoeiro, em monocultura e como componente de sistema agroflorestal. In: REUNIÃO NACIONAL DE PESQUISA DE FEIJÃO, 5., 1996, Goiânia. Anais... Goiânia: Embrapa-CNPAF; EMATER-GO, 1996. v. 1, p. 154-156.

GALVEZ, G. E.; GALINDO, J. J.; ALVAREZ, G. Desfoliación artificial para estimar perdidas por danos foliares en frijol (Phaseolus vulgaris L.). Turrialba, Costa Rica, v. 27, n. 2, p. 143-146, 1977.

HIGLEY, L. G. New understandings of soybean defoliation and their implication for pest Management. In: COPPPING, L. G.; GREEN, M. B.; REES, R. T. (Eds.). Pest management in soybean. London: Elsevier, 1992. p. 56-65.

HOHMANN, C. L.; CARVALHO, S. M. Efeito da redução foliar no rendimento do feijoeiro (Phaseolus vulgaris L.). In: REUNIÃO NACIONAL DE PESQUISA DE FEIJÃO, 1., 1982, Goiânia. Anais... Goiânia: EMBRAPA-CNPAF, 1982. p. 91-92. (Documento, 1).

JONES, L. H. Adaptative responses to temperature in dwarf french beans, Phaseolus vulgaris L. Annals of Botany, London, v. 35, p. 581-596, 1971.

LINK, D.; COSTA, E. C.; PANICHI, A. V. Efeito do desfolhamento artificial no rendimento de quatro variedades de feijoeiro (Phaseolus vulgaris L.). Revista do Centro de Ciências Rurais, Santa Maria, v. 4, n. 10, p. 329-333, 1980.

MOURA, G. de.; COSTA, J. A. Acúmulo de matéria seca em resposta ao desfolhamento na soja. Revista do Setor de Ciências Agrárias, Curitiba, v. 3, n. 1, p. 47-61, 1981.

MOURA, G. M. de; MESQUITA, J. H. de L. de. Influência do desfolhamento artificial no rendimento de grãos do feijoeiro (Phaseolus vulgaris), no Acre. In: REUNIÃO NACIONAL DE PESQUISA DE FEIJÃO, 1., 1982, Goiânia. Anais... Goiânia: EMBRAPA-CNPAF, 1982. p. 124-127. (Documento, 1).

NAKANO, O.; SILVEIRA NETO, S.; ZUCCHI, R. A Entomologia econômica. São Paulo: Ceres, 1981. 314 p.

OLIVEIRA, V. H.; ALVARENGA, M. I. N. Principais solos do Acre. Rio Branco: EMBRAPA-UEPAE, 1985. 40 p. (Documentos, 5).
PISSAIA, A.; COSTA, J. A. Influência de desfolhamentos artificiais sobre o rendimento de grãos e seus componentes, em duas cultivares de soja. Pesquisa Agropecuária Brasileira, Brasília, v. 16, n. 4, p. 507516, jul./ago. 1981.

PORTES, T. A. de. Ecofisiologia. In: ARAÚJO, R. S. Cultura do feijoeiro comum no Brasil. Piracicaba: POTAFOS, 1996. p. 101-137.

QUINTELA, D. E.; BARRIGOSSI, J. A. F. Resposta do feijoeiro à diferentes níveis de desfolha artificial. In: REUNIÃO NACIONAL DE PESQUISA DE FEIJÃO, 6., 1999, Salvador. Resumo... Santo Antônio de Goiás: EMBRAPA, 1999. p. 134-137. (Documentos, 99).

SANTA CECÍlIA, F. C.; RAMALHO, M. A. P.; GOMIDE, M. B. Efeito do desfolhamento sobre a produção de feijão (Phaseolus vulgaris L.). Ciência e Prática, Lavras, v. 4, n. 1, p. 66-72, 1980.

SANTOS, J. B. dos; GAVILANES, M. L. Botânica. In: VIEIRA, C.; TRAZILBO, J. P. J. de; BORÉM, A. Feijão: aspectos gerais e cultura no Estado de Minas. Viçosa: UFV, 1998. p. 56-81.

SCOTT, A. J.; KNOTT, M. A cluster analysis method for grouping means in the analysis of variance. Biometrics, Washington, v. 30, p. 507-512, 1974.

VALÉRIO, C. R.; ANDRADE, M. J. B. de; FERREIRA, D. F. Comportamento das cultivares de feijão Aporé, Carioca e Pérola em diferentes populações de plantas e espaçamentos entre linhas. Ciência e Agrotecnologia, Lavras, v. 23, n. 3, p. 515-528, jul./set. 1999.

WILCKEN, C. F.; MORAES, R. C. B.; HADDAD, M. L.; PARRA, J. R. P. Técnica de medição de área foliar consumida por insetos através de digitalizador de imagens acoplado a microcomputadores. In: CONGRESSO BRASILEIRO DE ENTOMOLOGIA, 15., 1995, Caxambu. Resumos... Caxambu: Sociedade Entomológica do Brasil, 1995. p. 39.

YOKOYAMA, L. P.; DEL PELOSO, M. J.; DISTEFANO, J. G.; YOKOYAMA, M. Nível de aceitabilidade da cultivar feijão "Pérola": avaliação preliminar. Santo Antônio de Goiás: EMBRAPA, 1999. 20 p. (Documentos, 98). 